\title{
Formation of Normal Gingival Epithelial Phenotypes Around Osseo- Integrated Oral Implants in Humans
}

\author{
Ian C. Mackenzie* and Maurizio S. Tonetti
}

THE ORAL, ORAL SULCULAR, AND JUNCTIONAL epithelia of the natural gingiva each possess distinct patterns of differentiation that are demonstrable both ultrastructurally and by their individual patterns of macromolecular synthesis. The supracrestal tissues reformed around oral implants structurally resemble those of natural gingiva but little is known about phenotypic changes occurring in the epithelia. To investigate whether peri-implant epithelia acquire similar patterns of differentiation to those of natural gingiva, biopsies from the supracrestal regions of five oral implants were examined by immunofluorescent methods using a panel of monoclonal antibodies with specificities for individual -cytokeratins and ICAM-1, macromolecules which act as markers of the three gingival epithelial phenotypes. The observed staining patterns indicated the formation of oral, oral sulcular, and junctional epithelia which were phenotypically indistinguishable from those of natural gingival epithelia. This degree of reprogramming of epithelial gene expression is a surprising observation and the potential mechanisms leading to the development of those new epithelial phenotypes are discussed in the context of what is known about the development of natural gingiva, in terms of the possible effects of inflammation, and in relation to the known connective tissue influences on epithelial differentiation. J Periodontol 1995;66:933-943.

Key Words: Dental implants; gingiva/anatomy and histology; epithelium/anatomy and histology; keratin.

Technical and clinical advancements in oral implants, mainly associated with successful manipulation of the osseous/implant interface, ${ }^{\prime}$ have led to the expectation of long-term maintenance of oral implants. ${ }^{2}$ These advances have increased the importance of secondary factors, such as infection of the marginal peri-implant tissues, which may be associated with the late failure of implants. ${ }^{3}$ Teeth are highly-evolved structures and one of their unique anatomical features is their "trans-epithelial" position: the crown of a tooth lies within the external environment of the oral cavity whereas the root lies embedded within the oral tissues. ${ }^{4}$ The epithelium, which elsewhere forms a continuous barrier between the internal and external environments, is thus disrupted and the gingival epithelia form a specialized cuff around the neck of the tooth. ${ }^{5}$ While this dento-gingival attachment generally provides an adequate functional seal, it forms a site that is prone

*Currently, School of Dentistry, University of Michigan, Ann Arbor; previously, Dental Branch, University of Texas Houston Health Science Center, Houston.

Department of Periodontology and Fixed Prosthodontics, School of Dental Medicine, University of Berne, Berne, Switzerland. to the inflammation and tissue breakdown associated with periodontal disease. ${ }^{6}$ Oral implants being similarly placed in a "trans-epithelial" position are also prone to inflammation of the marginal tissues and involvement in a process of tissue breakdown resembling that occurring around natural teeth. ${ }^{3}$

The ultrastructure of gingival epithelia of the normal human dentition was definitively described by Schroeder and Listgarten ${ }^{7}$ with the epithelia of this region being classified as 1) the junctional epithelium (JE), 2) the oral sulcular epithelium (OSE), and 3) the oral gingival epithelium (OGE). While the OSE and the OGE are similar, respectively, to non-keratinized and keratinized oral epithelia, the JE is a highly unusual epithelium. It lacks the distinctive patterns of maturation seen in the other oral epithelia and forms an attachment apparatus, consisting of a basal lamina and hemi-desmosomes, against the nonvital, calcified surface of the tooth. ${ }^{7}$

The molecular markers that characterize regionallyspecific patterns of differentiation in stratified squamous epithelia are typically expressed as newly-formed cells which emigrate from the basal into the suprabasal strata. ${ }^{8.9}$ 
Staining with antibodies directed against such differentiation products therefore provides a measure both of regional differences between epithelia and of the stage of differentiation of particular cells within an epithelium..$^{10}$ Distinct phenotypic differences between each of the three gingival epithelia have been demonstrated by such methods: some macromolecules are expressed by all three gingival epithelia but it has been found that each epithelial region expresses some markers not expressed by the other regions. ${ }^{11-14}$ The JE pattern of marker expression is primarily characterized by the absence of suprabasal differentiation markers typical of the other gingival epithelia and suprabasal expression of cell surface carbohydrates and cytokeratins that are characteristic of simple, rather than oral, epithelia. ${ }^{11,15}$ The JE also expresses various other markers, such as ICAM-1, transferrin receptors, and collagen VIII, that are either not expressed, or are expressed at lower levels, by the other oral epithelia. ${ }^{16}$ The precise significance of this unusual JE phenotype is as yet uncertain but it seems probable that it is related to various physiological aspects of sulcular function as well as to the maintenance of physical attachment to the tooth.

The apparent importance of this specialized dento-gingival attachment apparatus to the health of normal teeth has led to questions about the nature of the epithelial seal that is formed around implants. ${ }^{17-22}$ Attention has focused mainly on the anatomical structure of the "gingival" soft tissues reformed around the implant and on the development of an epithelial attachment to the implant surface. Histological and ultrastructural studies indicate that the superficial portion of the supracrestal tissues around an implant reorganize into a structure resembling normal gingiva and that the pattern of epithelial organization structurally matches that of the natural dentition: the oral epithelium is continuous with sulcular epithelium which is continuous, most apically, with junctional epithelium. There is some evidence that the patterns of differentiation of the epithelia formed around implants are similar to those of normal gingiva. ${ }^{23}$ Several studies have shown that epithelium attaches to the surface of metallic, ceramic, and other types of implant materials with the development of a basal lamina and hemi-desmosomes similar to those present at the dento-gingival junction. ${ }^{24-27}$

Normally, when the surface continuity of an epithelium is disrupted, lateral migration of epithelial cells at the wound margin is activated ${ }^{28}$ and ceases only when the free edges of epithelium meet and continuity is restored. ${ }^{29}$ It seems to be generally assumed that the "free edge" of junctional epithelium that forms around a normal tooth is prevented from migrating beyond the cemento-enamel junction by the cementum or the density of the periodontal ligament fibers inserted into it: in the absence of similar fiber insertion into the surface of an implant, it has been questioned why the epithelium around an implant does inot migrate apically along the implant surface, at least to the level of osseointegration. ${ }^{18-20}$ It has previously been suggested that simple "physical" obstruction of an epithelium by collagen fibers would by itself be inadequate to prevent apical migration of epithelium and that an intrinsically passive or non-migratory property may be associated with the unusual phenotype expressed by junctional epithelium. ${ }^{30,31}$ Apical migration of the junctional epithelium does of course occur in periodontal disease in association with inflammation and loss of attachment but the mechanism of this event is unclear. The patterns of epithelial differentiation that develop around an implant therefore appear to be of interest, first because these structures form in the absence of either odontogenic epithelium or periodontal ligament, which have been implicated in the formation of normal $\mathrm{JE}, 5,7,30$ and second, because comparison of the gingival epithelia of normal teeth with that of implants might be of value in understanding normal gingival function.

\section{MATERIALS AND METHODS}

\section{Biopsies}

The tissues examined in the present study were part of a series of biopsies taken originally to study the expression of vascular adhesion molecules. ${ }^{32}$ These biopsies were obtained, with informed consent, from systemically healthy volunteers. The implants for biopsy were selected according to the clinical criteria of 1) absence of detectable bacterial plaque accumulation; 2) probing depths $<3 \mathrm{~mm}$; and 3) absence of suppuration or bleeding on probing. The biopsies were taken from around the margins of transmucosal titanium implants $\ddagger$ to 9 months following implantation in partially edentulous individuals. Achievement of osseointegration was verified according to clinical and radiographic criteria. Each subject contributed one biopsy which was immediately embedded and snap frozen in liquid nitrogen. Frozen sections were cut in a cryostat at $6 \mu \mathrm{m}$, air-dried overnight, and briefly prefixed in acetone. Only sections that showed crestal and sulcular epithelia cut in the appropriate plane were included in the study. The results presented here are based on the examination of biopsies of tissues around implants of five separate patients. These were compared with the results for biopsies of clinically-uninflamed gingiva from the buccal surface of nine natural human teeth which were collected and similarly processed as previously described. ${ }^{11,15}$ Specimens were collected according to a protocol approved by the institutional Committee for Protection of Human Subjects.

\section{Immunohistochemical Procedures}

Sections of each implant and natural gingival specimens were stained using a panel of antibodies with defined

FITI Bonefit, Straumann Institute, Waldenburg, Switzerland. 

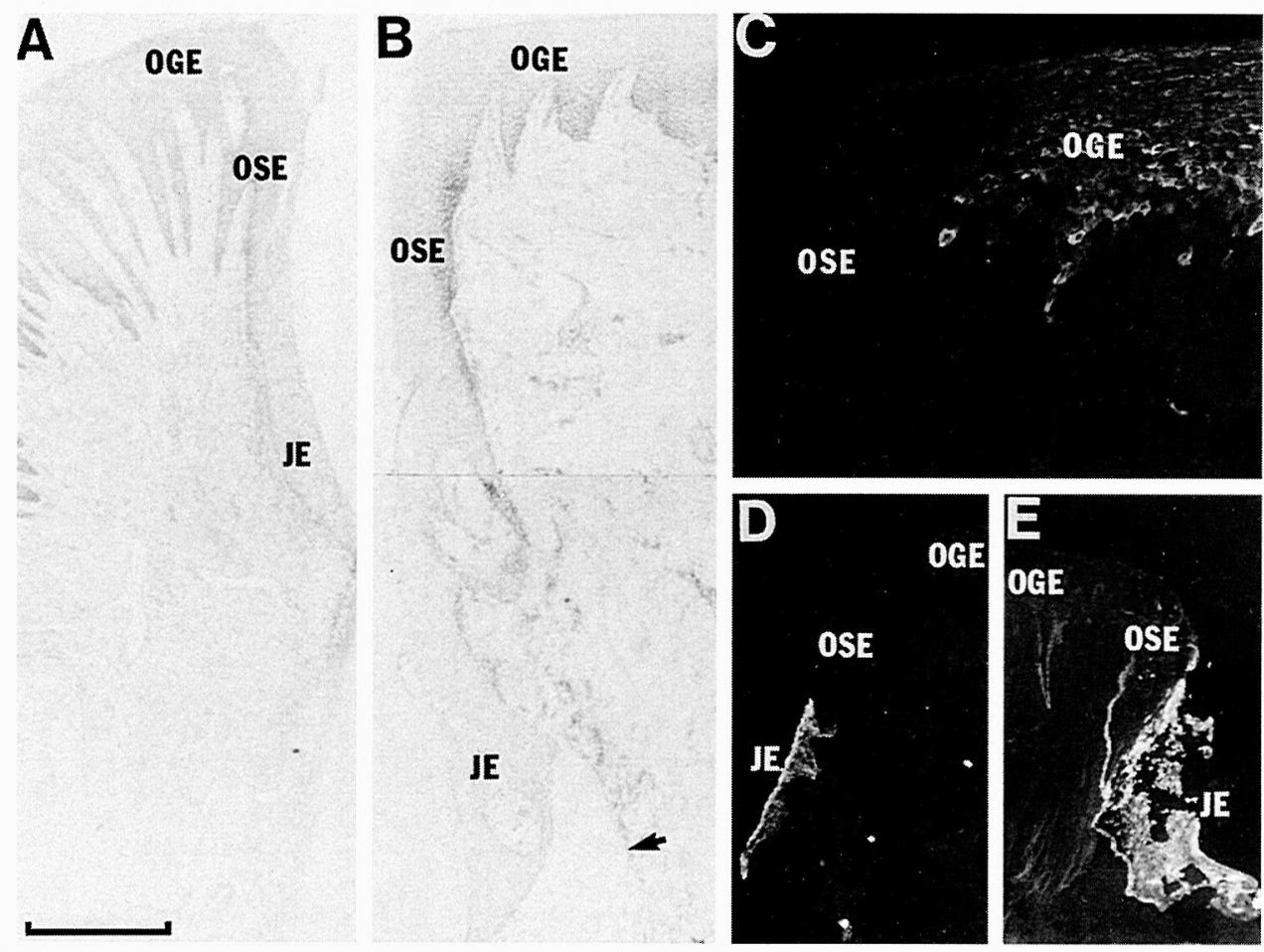

Figure 1. Sections of natural $(\boldsymbol{A})$ and implant $(\boldsymbol{B})$ gingiva stained with hematoxylin to show general structure. Both tissues show low levels of inflammation but the implant gingiva shows a greater length of OSE and a thicker and less regular JE which, apically, has artifactually separated from the CT (arrow). Immunofluorescent staining of the region of junction between the OGE and OSE of implant gingiva for $K 10(C)$ shows expression restricted to the suprabasal cells of the OGE. Staining for K19 in both natural (D) and implant (E) gingiva shows strong reactivity in all strata of the JE. The implant gingiva also shows some staining of basal cells of the OSE and OGE, a pattern found in inflamed specimens of natural gingiva (scale for $A, B$ $\simeq 0.4 \mathrm{~mm}$, for $C=100 \mu \mathrm{m}$, and for $D, E=0.6 \mathrm{~mm}$ ).

specificities for individual cytokeratins, for ICAM-1 and for laminin as listed in Table 1. Information about the sources and specificities of these antibodies, and the details of the staining methods used, have been published previously. ${ }^{11,16}$ In brief, sections were stained by applying each mAb, usually as the undiluted culture supernatant, to sections that were then incubated overnight in a moist chamber at $4^{\circ} \mathrm{C}$. After washing in three changes of phosphate-buffered saline, pH 7.4 (PBS), over 15 minutes, $\mathrm{mAb}$ binding was visualized using second-layer antibodies against mouse or rat immunoglobulins, as appropriate to the primary $\mathrm{mAb}$, conjugated to fluorescein isothiocyanate. ${ }^{\S}$ Sections were incubated with second-layer antibodies at a dilution of 1:80 in PBS and were mounted in buffered glycerin ( $\mathrm{pH}$ 7.4) containing paraphenylenediamine as an anti-fading agent. ${ }^{15}$ Control reactions for the specificity of staining of each of the primary antibodies consisted of staining oral mucosa with the secondlayer conjugate alone and substitution of the primary $\mathrm{mAb}$ with mouse mAbs of known irrelevant specificities. Comparison of the staining patterns for each different

sakopatts, Copenhagen, Denmark.
$\mathrm{mAb}$ within each specimen provided an additional internal control for confirmation of specificities.

\section{RESULTS}

With routine histological staining, each of the implant biopsies examined showed a structure that was basically similar to that of normal human gingiva (Figs. 1A and 1B). The oral aspect of the biopsies was covered with a differentiating epithelium with well-formed rete, corresponding to the OGE of normal gingiva, which was continuous with an epithelium of similar histological appearance, but with few rete, which extended for a variable distance down into the sulcular region. This epithelium, corresponding to the OSE of normal gingiva, was continuous apically with a hypochromatic epithelium, typically with a smooth epithelial-connective tissue interface (Fig. 1B), that showed little sign of differentiation. The extent to which the full length of this epithelium, which corresponded to the JE of normal gingiva, was included in the biopsies could not be ascertained but, in several sections, the most apical part of the epithelium had artifactually separated from the connective tissue (Fig. 1B). Although the basic patterns of epithelial organization 
Table 1. Comparison of Patterns of Cytokeratin Expression in the Oral Gingival (OGE), Oral Sulcular (OSE) and Junctional (JE) Epithelia Around Teeth and Around Implants.

\begin{tabular}{|c|c|c|c|c|c|c|}
\hline & \multicolumn{2}{|c|}{ OGE } & \multicolumn{2}{|c|}{ OSE } & \multicolumn{2}{|c|}{ JE } \\
\hline & Implant & Tooth & Implant & Tooth & Implant & Tooth \\
\hline \multicolumn{7}{|c|}{ All stratified epithelia } \\
\hline K5 (Basal 2) & + & + & + & + & + & + \\
\hline K14 (LL001) & + & + & + & + & + & + \\
\hline \multicolumn{7}{|l|}{ Cornified epithelia } \\
\hline K1 (polyclonal) & + & + & - & - & - & - \\
\hline K10 (RKSE60) & + & + & - & - & - & - \\
\hline \multicolumn{7}{|c|}{ Non-cornified epithelia } \\
\hline $\mathrm{K} 4(6 \mathrm{~B} 10)$ & +- & +- & + & + & +- & - \\
\hline $\mathrm{K} 13(1 \mathrm{C} 7)$ & - & - & + & + & + & + \\
\hline \multicolumn{7}{|l|}{ Simple epithelia } \\
\hline K19 (LP2K) & - & - & +- & +- & + & + \\
\hline K8 (Troma 1) & - & - & - & - & +- & + \\
\hline K18 (LE 61) & - & - & - & - & +- & + \\
\hline ICAM-1 (CD54) & - & - & - & - & + & + \\
\hline Laminin & + & + & + & + & +- & + \\
\hline
\end{tabular}

The first column indicates the individual cytokeratins examined, the type of epithelium in which they are normally expressed, and the monoclonal antibodies used (see text). Data are also included for the adhesion molecule ICAM-1 and for laminin, a basal lamina component. Staining patterns indicated as either + or are those that appeared consistent for each of the specimens examined. Staining patterns indicated as +are those that showed, as described in the text, variation within each epithelial region or from one specimen to another.

were similar for the tooth and the implant biopsies, the implant specimens tended to show a greater length of epithelium abutting the implant surface than was found abutting the tooth surface. This was due partly to a more apical extension of the OSE and partly to a more extensive $\mathrm{JE}$ which was also usually thicker than that of natural gingiva.

Immunohistochemical staining demonstrated three distinct epithelial phenotypes in both natural and implant gingiva and, apart from minor differences of intensity of the staining reactions, the findings were consistent for all of the specimen examined. The staining patterns observed are summarized in Table 1 . For $\mathrm{Kl}$ and $\mathrm{K} 10$, the cytokeratins typically expressed as differentiation markers in cornifying epithelia, both natural and implant gingiva showed suprabasal staining of the OGE (Fig. 1C), but not of the OSE or JE. Natural and implant gingiva also showed a similar pattern of staining of the junctional epithelium for $\mathrm{K} 19$, a keratin that is typically found in simple epithelia and, variably, in basal cells of oral epithelia (Figs. 1D and 1E). All strata of the JE were strongly stained but neither the implant or natural gingiva showed staining for K19 in the OGE or OSE except in patches of basal cells. Staining for K8 and K18 (Figs. 2A and 2B), cytokeratins that are expressed in simple epithelia, but not in oral epithelia except in Merkel cells, was absent from OGE and OSE of implant and natural gingiva. The $\mathrm{JE}$ of natural gingiva showed variable but moderately strong staining of suprabasal cells for $\mathrm{K} 8$ and $\mathrm{K} 18$ and similar, but somewhat weaker, staining was also seen in the $\mathrm{JE}$ formed around implants. In the OGE of both nat- ural and implant gingiva, elliptical cells staining strongly for $\mathrm{K} 8$ and $\mathrm{K} 18$, presumably Merkel cells, were present as individual cells or clusters at the tips of the rete. An unusual feature of the implant gingiva was that such cells were also occasionally seen in the OSE region.

Both natural and implant gingiva showed similar patterns of staining for $\mathrm{K} 4$ and $\mathrm{K} 13$, the cytokeratins expressed as differentiation markers in non-cornifying epithelia (Fig. 2C through F). Strong staining of all suprabasal cells for $\mathrm{K} 4$ was present in the OSE region and there was staining of scattered individual cells in the adjacent region of the OGE. The natural gingival specimens typically showed a sharp line of demarcation between the OSE cells, which stained for $\mathrm{K} 4$, and the JE cells which were unstained. However, the implant specimens showed a less precise demarcation with staining of scattered cells in the coronal part of the JE. In natural gingiva there was strong staining for $\mathrm{K} 13$ in the OSE region and strong, but somewhat less intense, staining of the JE. A similar pattern of staining of the OSE and JE was seen in implant gingiva. Staining for K5 and K14, the pair of the cytokeratins typically expressed by basal cells of all stratifying epithelia, was seen in all epithelial regions of both implant and natural gingiva. In both implant and natural gingiva, staining for K5 was seen throughout the full thickness of the JE whereas staining was restricted to the basal and parabasal cells of OSE and OGE.

In natural human gingiva, ICAM-1 is expressed on the surface of JE cells but not by OSE or OGE and an essentially similar pattern of staining was seen in implant gingiva (Fig. 3). In the OGE region, the epithelium was 

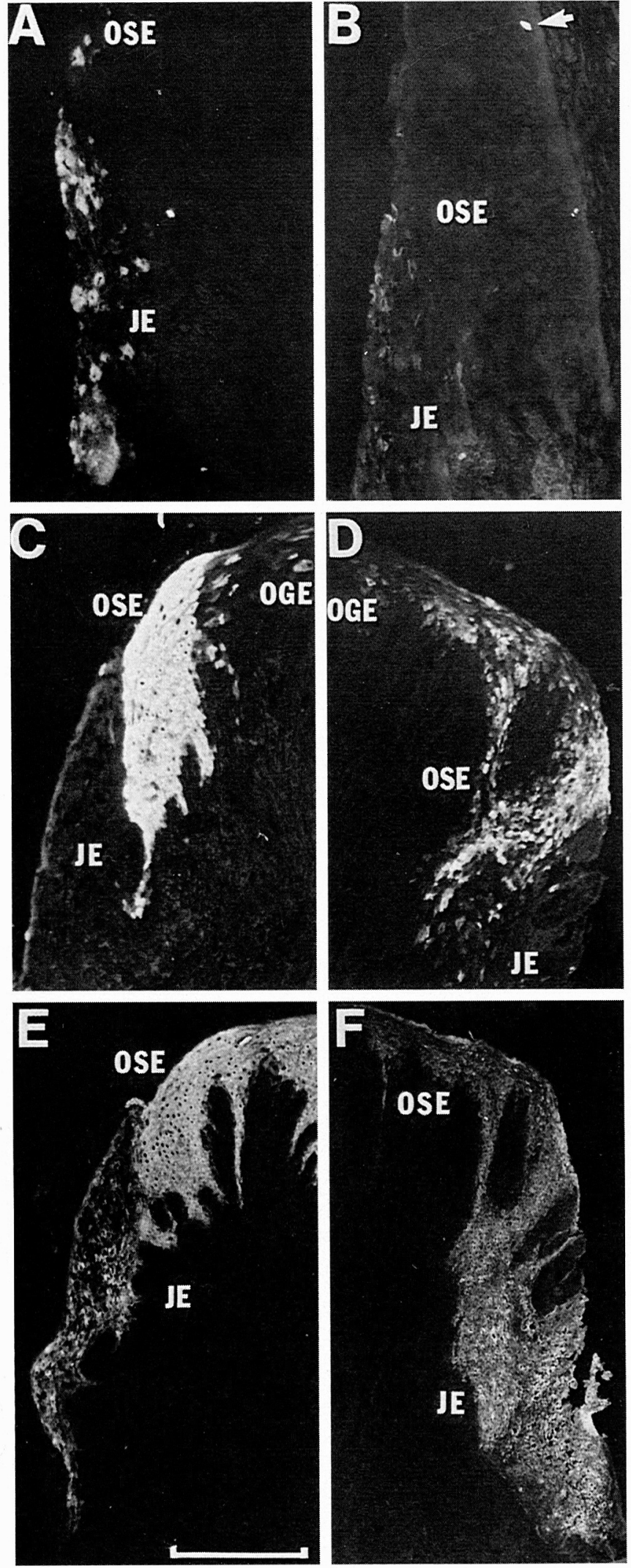

unstained but weak staining was present on the surface of endothelial cells of the sub-epithelial capillaries. Typically, staining was absent from the OSE but, in some regions where there appeared to be sub-epithelial collections of inflammatory cells, staining of the epithelium was present and was stronger on the basal than the suprabasal cells. All cells of the JE of the implant specimens showed some staining for ICAM-1 with the strongest staining seen for suprabasal cells in the coronal region. Typically there was a clear line of demarcation between the strongly stained JE and the unstained OSE (Fig. 3C).

The epithelial-connective tissue interface of all regions of natural and implant gingiva showed staining for laminin (Fig. 4). In the OGE and OSE regions there was smooth continuous staining of the basement membrane zone and staining of the sub-epithelial capillaries. Beneath the coronal region of the JE there was clear staining of the basement membrane but there were strongly stained irregular structures lying close to, or in contact with, the basement membrane. These structures appeared to be capillaries but were less regularly organized than in the OGE and more closely associated with epithelial basement membrane. In the apical region of the JE there was often weaker and patchy staining for laminin.

\section{DISCUSSION}

Previous histological and ultrastructural studies have shown that the "gingiva" formed from the alveolar mucosa around dental implants is anatomically similar to the gingiva around normal teeth. ${ }^{19-21}$ The present study indicates that this anatomical rearrangement is associated with new patterns of epithelial gene expression that lead to phenotypic patterns characteristic of the JE, OSE, and OGE of natural gingiva. Although relatively few specimens were examined, the consistency of the basic marker patterns found in each of the specimens of implant gingiva examined allows confidence in this conclusion. Except for some minor differences in the sharpness of demarcation of one region from another, natural and implant gingival epithelia were basically indistinguishable in terms of the cytokeratins used as phenotypic markers. The

Figure 2. Immunofluorescent staining of natural $(A, C, E)$ and implant $(B, D, F)$ gingiva for $K 18, K 4$, and $K 13$. Natural gingiva shows moderate staining of suprabasal JE cells for $K 18(\boldsymbol{A})$ and a similar but weaker staining pattern is seen in the implant gingiva $(\boldsymbol{B})$ which also shows a strongly stained elliptical cell, probably a Merkel cell, in the basal region of the OSE (arrow). Strong staining for $K 4$ is restricted to suprabasal cells of the OSE in both natural $(C)$ and implant gingiva $(D)$. Both of these specimens show only a short length of OSE with some extension of scattered stained cells into the OGE region. There is a sharp demarcation between JE and OSE in the natural gingiva but the implant gingiva shows a less sharp demarcation with some scattered stained cells in the coronal part of the JE. Staining for K13 is seen in suprabasal cells of the JE and OSE of both natural $(E)$ and implant $(F)$ gingiva (scale for $A, B=0.2 \mathrm{~mm}$, for $C, D=0.3 \mathrm{~mm}$ and for $E, F=0.5 \mathrm{~mm}$ ). 

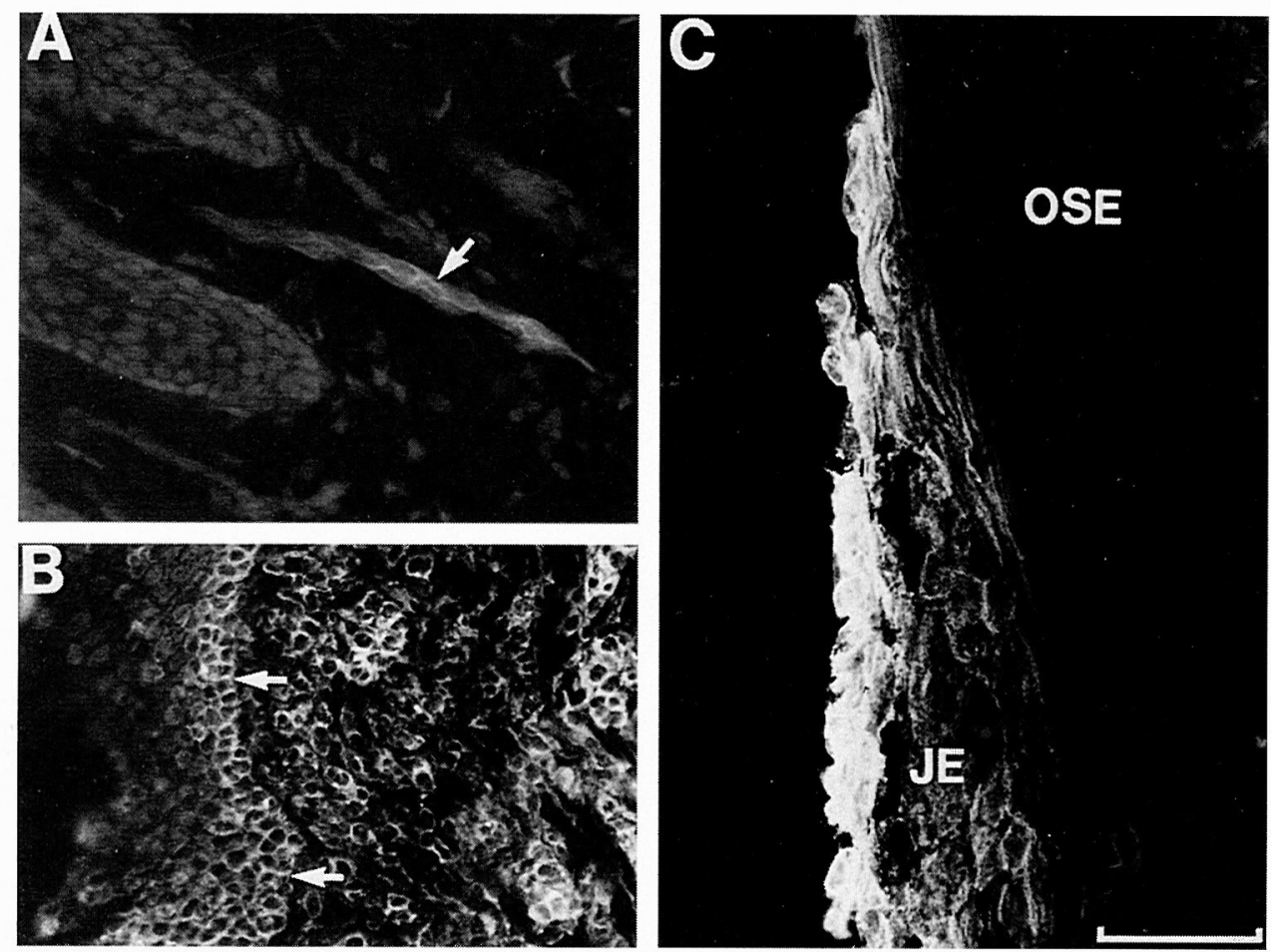

Figure 3. Implant gingiva stained for ICAM-1. In the OGE region (A) there is no epithelial staining but weak staining of capillary endothelium is seen (arrow). Typically, staining for ICAM-I was absent from the OSE but inflamed specimens of OSE (B) showed staining of (inflammatory) cells in the connective tissue and quite strong staining of basal and parabasal epithelial cells (arrows). The JE of all specimens showed staining for ICAM- 1 which was strongest coronally and towards the tooth surface $(C)$ and there was typically a clear line of demarcation between the JE and the sustained OSE (scale for $A=100 \mu \mathrm{m}$, for $B=150$ $\mu \mathrm{m}$, and for $C=120 \mu \mathrm{m})$.
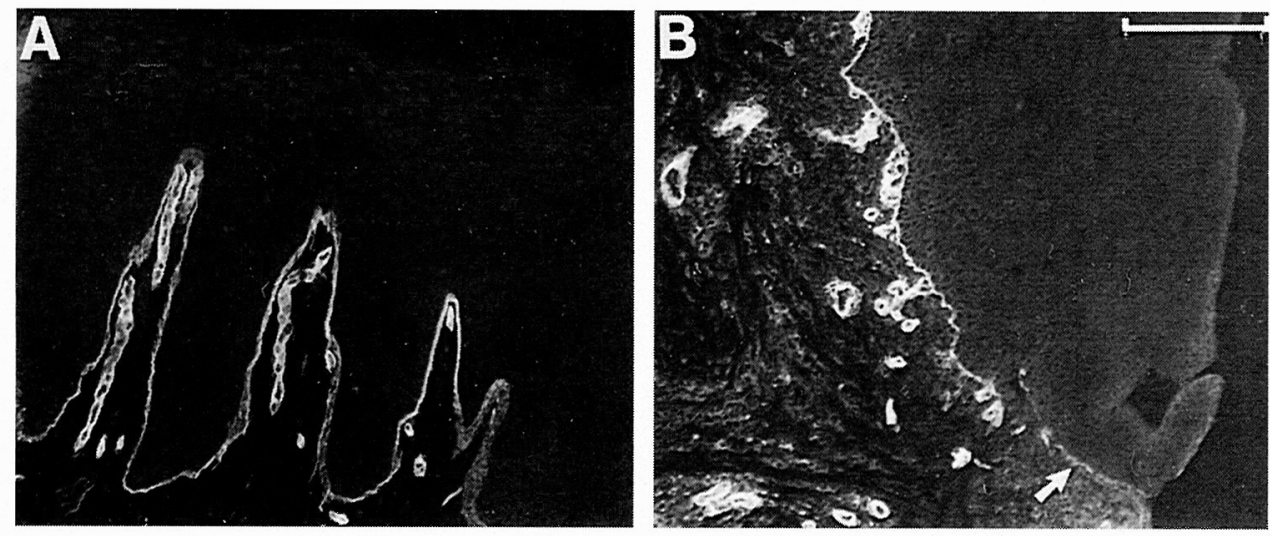

Figure 4. Immunofluorescent staining of the OSE $(\boldsymbol{A})$ and the JE $(\boldsymbol{B})$ regions of implant gingiva for laminin. Clear staining of epithelial and capillary basal lamina is seen in the OSE and JE. However, the JE shows close epithelial approximation of irregularly disposed capillaries and weaker staining (arrow) for epithelial basal lamina in the apical region (scale $=100 \mu \mathrm{m})$.

JE around implants also expressed ICAM-1, an additional phenotypic marker of the JE of natural teeth. ${ }^{16,33}$ The control specimens of natural gingiva were not matched for age or sex with the implant specimens as previous studies have shown that gingival epithelial differentiation is not significantly altered by these parameters. Inflammation has not been found to significantly affect the basic pattern of differentiation of gingiva ${ }^{34}$ and the inflammatory status of the implant and gingival specimens was not clinically assessed in detail as it could be subsequently assessed from the tissue sections.

In view of the usual phenotypic stability of adult epithelia, the acquisition of new phenotypes by the mucosal epithelium surrounding an implant is an interesting ob- 
servation, but one that is rather difficult to explain. This difficulty is compounded by the present lack of information about the factors that control epithelial differentiation and function around a natural tooth. Ten Cate ${ }^{17}$ discussed the formation of gingival epithelial anatomy around implants within the context of basic patterns of epithelial behavior and the development of the dentogingival junction of natural teeth. His observations are extended by those of the present study and it appears that the "experiment" of placing an implant through the oral mucosa points to some interesting conclusions. First, although the JE formed during the eruption of natural teeth is probably derived from cells of the reduced enamel epithelium, ${ }^{7}$ its presence around implants indicates that its formation does not require the participation of odontogenic epithelium: this supports conclusions previously drawn from the observed reformation of JE following gingivectomy ${ }^{35,36}$ Second, the presence of JE and OSE phenotypes around an implant suggest that these epithelial structures do not represent a specifically "dental" phenomenon: neither particular properties of a tooth, as such, nor of periodontal ligament connective tissue are necessary for their formation. Third, the reformation of gingival epithelia around an implant is associated with reprogramming of the basic patterns of epithelial macromolecular synthesis and these are probably of functional significance. However, the relevant functional attributes of these epithelial phenotypes remain to be elucidated. Finally, although the expression of the various markers examined indicate a marked similarity, it remains uncertain whether the epithelial phenotypes formed around implants are identical in all respects to those of natural teeth.

Developmental interactions between an epithelium and its underlying connective tissue essentially determine the phenotype that it expresses throughout adult life but various factors such as wounding, inflammation, and other pathological changes can subsequently modify its basic pattern of differentiation. ${ }^{37-39}$ Wounding activates migratory behavior and alters patterns of epithelial differentiation and, in the epidermis for example, wounding induces expression of $\mathrm{K} 19$, and of $\mathrm{K} 6$ and $\mathrm{K} 16$, keratins that are considered markers of hyperproliferation in epidermis but are normally expressed by mucosal epithelia..$^{40,41}$ Some parallels can also be drawn between the patterns of integrin expression in wounded epidermis ${ }^{42}$ and in normal human gingiva ${ }^{43}$ However, although wounding has been shown to lead to loss of some of the normal differentiation markers of mucosal epithelia, ${ }^{29}$ as yet, little is known about the general effects of wounding on the mucosal epithelial phenotype.

Ten Cate ${ }^{17}$ has suggested that connective tissue inflammation determines the character of the sulcular epithelium and, in part, the anatomy of the JE. Inflammation is known to alter the normal patterns of keratin expression of gingival epithelia: ${ }^{38}$ in particular, it increases the ex- pression of $\mathrm{K} 4$ and $\mathrm{K} 13$, the keratins typical of the OSE phenotype, and leads to the basal, and some suprabasal, expression of K19, a keratin strongly expressed both basally and suprabasally by the JE. Similar changes in keratin expression are also seen in the oral epithelium growing into the openings of sinus tracts associated with periapical lesions. ${ }^{44}$ Direct comparison of the implant biopsies with the biopsies from natural teeth should be treated with caution, as the latter were taken from newly-erupted teeth selected for the absence of clinical inflammation. Possibly, small anatomical differences, such as the tendency to a greater depth of the sulcular region around implants than natural teeth, observed in this and some other studies, ${ }^{19}$ could be a result of previous or persistent low levels of inflammation. ${ }^{32}$ Similarly, the observation of a less precise demarcation between JE, OSE, and OGE phenotypes could be an effect of inflammation as inflamed human periodontal pockets may show some apical encroachment of the OSE and some loss of the sharpness of demarcation between the JE, OSE, and OGE. ${ }^{34}$ However, the same basic anatomical distribution of JE, OSE, and OGE is maintained and other observations also suggest that although inflammation may lead to sulcus deepening, it may not be a major factor in determining the basic patterns of gingival epithelial differentiation. In rodents, for example, the JE, OSE, and OGE have a distinct anatomical arrangement that is demonstrable in both normal and germ free animals. ${ }^{45,46}$ These epithelia have simple, noncornified and cornified patterns of keratin expression that parallel those in human gingiva ${ }^{30,31}$ and these patterns of keratin expression are essentially similar in germ-free rats (unpublished observations). Nevertheless, as Schroeder and Listgarten ${ }^{7}$ and Ten Cate ${ }^{17}$ have pointed out, reduced levels of gingival inflammation are associated with loss of epithelium with the typical appearance of OSE and the observations of Bosch et al..$^{38}$ indicate that inflammation reduces expression of $\mathrm{K} 1$ and enhances expression of $\mathrm{K} 4$ and $\mathrm{K} 13$ in gingival epithelium. It thus appears to produce a shift from the OGE towards the OSE phenotype. Possibly, therefore, inflammation is associated with the expression of the OSE phenotype in both natural human gingiva and around implants.

The JE phenotype does not correspond to that of any other oral epithelium. Structurally the JE is a stratifying epithelium and it expresses $\mathrm{K} 5$ and $\mathrm{K} 14$, keratin markers that are typical of stratifying epithelia. Its unusual features are its suprabasal expression of K8 and K18, keratins typically expressed only by simple epithelia, and its basal and suprabasal expression of $\mathrm{K} 19$, a transitional keratin ${ }^{9}$ that is typically expressed by simple epithelia and basally by some stratifying epithelia. ${ }^{12}$ Co-expression of simple and stratifying epithelial keratins occurs in odontogenic epithe$\mathrm{lia}^{47}$ and is common in various cysts derived from odontogenic epithelia. ${ }^{48}$ It does not typically occur in adult oral mucosal epithelia but simple epithelial keratins are nor- 

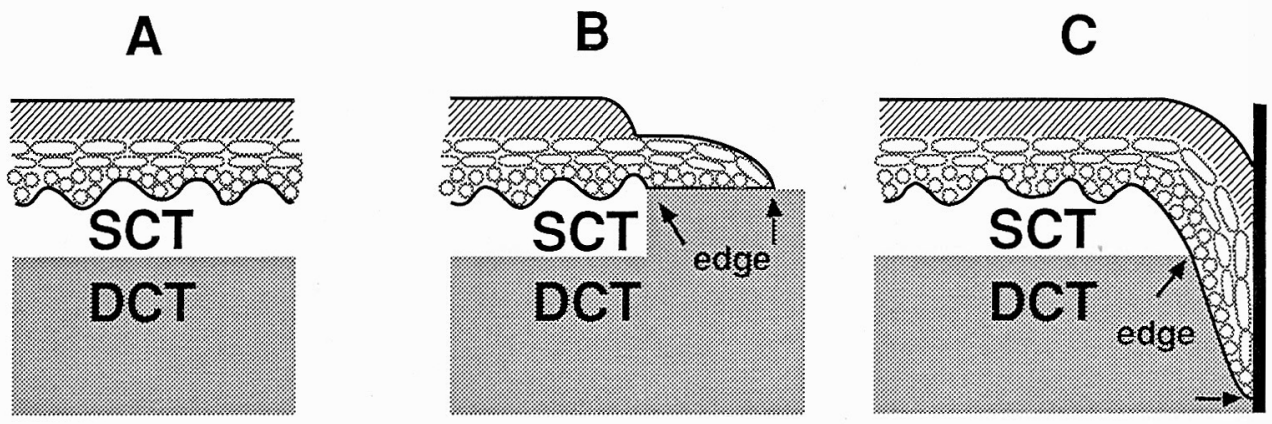

Figure 5. Diagram of the postulated relationships of epithelium to superficial and deep connective tissues in normal tissue $(\boldsymbol{A})$, in experimental mucosal transplants $(\boldsymbol{B})$, and around implants $(\boldsymbol{C})$. In normal mucosa $(\boldsymbol{A})$, the epithelium is supported by a layer of sub-epithelial connective tissue (SCT) which separates it from the deeper connective tissue (DCT). The SCT provides permissive stimuli for normal epithelial growth and differentiation and differs from DCT which lacks such stimuli. When pieces of full thickness mucosa are transplanted to DCT sites $(\boldsymbol{B})$, a junction is formed between the transplanted SCT and the DCT of the graft bed. The epithelium differentiates normally-over its transplanted SCT but at the edge of the transplant, where epithelium is brought artificially into contact with DCT, it shows abnormal migration and differentiation due to a lack of permissive stimuli from DCT. A small edge of epithelium grows a short distance onto the DCT but further outgrowth does not occur and this edge of epithelium (between arrows) shows mixed expression of stratifying and simple epithelial keratins. ${ }^{30.64} A$ similar unusual epithelial-connective tissue relationship results from implant placement $(C)$. The presence of the implant prevents the normal healing pattern of epithelial migration to close the surface of the wound. Instead, epithelial migration is deflected down the interface between the implant and the SCT. When the epithelium grows down beyond the SCT to reach the DCT (between arrows) its relationship to SCT and DCT is similar to that shown in panel $B$ and its acquires the "edge" pattern of differentiation. The unusual co-expression of simple and stratifying epithelial keratins seen in JE corresponds to the "edge" phenotype and it is assumed that JE therefore acquires the same stable, non-migratory character as edge epithelium.

mally expressed by the oral epithelia during development ${ }^{49}$ and such expression is maintained or acquired in specialized epithelial structures such as taste buds. ${ }^{50}$ Simple epithelial keratins are also expressed by the stratifying epithelia of oral leukoplakias and squamous cell carcinomas. ${ }^{37}$ However, apart from demonstrating that mucosal epithelia have the capacity to express simple epithelial keratins, these observations do not appear to explain how such expression is acquired by the JE that is formed around implants. Inflammation does not appear to induce expression of simple epithelial keratins in oral epithelia ${ }^{38}$ and a structure similar to JE can be experimentally produced in the absence of inflammation in vitro. ${ }^{51}$ Inflammation may induce expression of ICAM-1 ${ }^{52}$ but is reported to induce ICAM-1 expression primarily on basal cells, ${ }^{53}$ as seen on the OSE in the present study, rather than suprabasally, as seen in the JE. Further, it appears that although the JE expression of ICAM-1 can be modified by inflammation, ${ }^{53,54}$ it is constitutively expressed by uninflamed JE both in vivo ${ }^{16}$ and in vitro. ${ }^{41}$

The essential roles of epithelial-mesenchymal interactions during embryonic development have been extensively studied. It is apparent that cascades of reciprocal interactions occur between epithelium and mesenchyme and, as a result of regionally-specific mesenchymal signals, an adult epithelium acquires a stable regionally-specific phenotype. ${ }^{39}$ However, adult tissues retain some capacity to generate and respond to further epithelial-mes- enchymal interactions: for example when epithelia are recombined with connective tissue from another region, they acquire new patterns of differentiation that are appropriate to the region of origin of the connective tissue. ${ }^{39,55}$ Such interactions are considered "directive" in that the connective tissue specifies a particular pattern of differentiation which the epithelium can then express, for example in vitro, in the absence of further regionally specific signals from its connective tissue. ${ }^{56}$ In the present studies, implants were placed through the mucosa of the edentulous alveolar ridge which is typically covered by a cornified epithelium corresponding to that of the OGE surrounding the implant. Possibly placement of an implant could generate additional types of directive signals from the alveolar mesenchyme that specify the new patterns of OSE and JE differentiation but the mechanism by which this could occur is unknown.

There are, however, other types of mesenchymal influences that are not of a directive nature and have been termed "permissive." 57 For example, embryonic epidermis or endoderm fails to differentiate if isolated from its mesoderm but normal development may be supported by recombining it with mesoderm from other regions. ${ }^{58,59}$ It has also been shown that embryonic mesenchymes differ in the degree to which they are able to provide such permissive influences. ${ }^{60}$ There is evidence that similar permissive interactions are necessary for the maintenance of epithelial differentiation in adult tissues and, in partic- 
ular, that superficial and deep connective tissues differ in their abilities to support epithelial growth and differentiation. For example, when adult epithelial cells are isolated and grown in vitro, they typically fail to express fully their in vivo phenotype but may do so if they are cocultured with dermal, but not deep, connective tissues. ${ }^{61}$ Work with both human and murine tissues suggests that dermal fibroblasts produce a diffusible stimulus to differentiation. ${ }^{61-63} \mathrm{~A}$ good demonstration of the differing abilities of deep and subepithelial connective tissues to support the growth of either murine and human epithelia is seen when full thickness oral mucosa is transplanted to deep connective tissue sites in vivo. ${ }^{31,64,65}$ The epithelium differentiates normally over its transplanted connective tissue, but it fails to show either normal migration or differentiation on the surrounding deep connective tissue. Limited migration onto the deep connective tissue around the transplant leads to a small, stable outgrowth of epithelium which is stratified and maintains expression of $\mathrm{K} 5$ and $\mathrm{K} 14$, the basic stratifying epithelial markers, but lacks the differentiation pattern of the normal epithelium. However, it shows suprabasal expression of simple epithelial keratins and thus acquires a phenotype similar to that of junctional epithelium. ${ }^{30,31}$

It thus appears that "subepithelial" and "deep" connective tissues differ functionally in their abilities to support epithelial growth and differentiation and recent work suggests that this difference may be due to the expression of stimulatory cytokines such as keratinocyte growth factor. 66,67 Further, the absence of such permissive stimuli from deep connective tissues leads to failure of epithelial migration and acquisition of a new "default" phenotype which corresponds to that of junctional epithelium. This behavior may at least partially explain development of the JE phenotype around implants. Figure 5 compares the relationships of epithelium to superficial and deep connective tissues in normal tissues, in experimental mucosal transplants, and in implants. It can be seen that the normal wound healing response of lateral migration of epithelium to restore epithelial continuity is prevented by the presence of an implant. Instead, the epithelium can only migrate down the connective tissue surface adjacent to the implant and in so doing, migrates beneath the zone of connective tissue permissiveness to reach the deep connective tissue. Here, due to the absence of permissive stimuli, it acquires the "edge" phenotype described above which is associated with stable non-migratory behavior and expression of markers similar to natural JE.

Previous work has shown that the intrinsic biological properties of epithelium enable it to attach to a wide variety of materials and that the oral mucosa can establish a satisfactory functional attachment to oral implants. ${ }^{17}$ The present work shows that implant placement is additionally associated with the development of new epithelial phenotypes with a spatial distribution that closely corre- sponds to that of natural gingiva and the formation of JE around the implant which is similar to the non-migratory "edge" of epithelium formed in experimental transplants to deep connective tissue. The latter observation appears to answer the question of why there is only limited migration of epithelium down an implant/connective tissue interface despite the absence of physical obstruction by collagen. ${ }^{20,30,31,68}$ It would also make it unnecessary to postulate "connective tissue integration" to prevent epithelial migration. ${ }^{19}$ The conclusion that permissive interactions are necessary for epithelial migration and differentiation would also appear to be of interest to the progression of periodontal disease around both implants and natural teeth. Currently, apical migration of epithelium seems to be considered a passive event that invariably follows destruction of connective tissue attachment to the tooth. However, if the above interpretation of $\mathrm{JE}$ behavior is substantiated, destruction of connective tissue attachment would not by itself lead to epithelial migration: generation of additional specific epithelial activation factors, possibly components of the inflammatory response, would be necessary for this component of disease progression.

\section{Acknowledgments}

We gratefully acknowledge the highly skilled assistance of Gillian Rittman with the immunofluorescent procedures. This work was supported in part by NIH NIDR grant R01 10317.

\section{REFERENCES}

1. Branemark P, Zarb G, Albrektsson T. Tissue Integrated Prostheses. Osseointegration in Clinical Dentistry. Chicago: Quintessence; 1985.

2. Albrektsson T, Dahl E, Enbom L, et al. A Swedish multicenter study of 8139 consecutively inserted Nobelpharma implants. J Periodontol 1988;59:287-296.

3. Tonetti MS, Schmid J. Pathogenesis of implant failures. Periodontology 2000 1994;4:127-138.

4. McKinney RV, Steflik DE, Koth DL. Per, peri, or trans? A concept for improved dental implant terminology. J Prosthet Dent 1984;52: 267-269.

5. Ten Cate AR. Oral Histology, Development, Structure and Function, 4th ed. St. Louis: The C.V. Mosby Company; 1994:268-274.

6. Page RC, Schroeder HE. Periodontitis in Man and Other Animals. A Comparative Review. Basel: Karger; 1982.

7. Schroeder HE, Listgarten MA. Fine structure of the developing epithelial attachment of human teeth. In: Wolsky A, ed. Monographs in Developmental Biology, vol. 2. Karger: Basel; 1977.

8. Fuchs E. Epidermal differentiation. Cell Biol Int 1990;2:1028-1035.

9. Purkis PE, Steel JB, Mackenzie IC, Nathrath WJB, Leigh IM, Lane EB. Antibody markers of basal cells in complex epithelia. $J$ Cell Sci 1990;97:39-50.

10. Morgan PR, Leigh IM, Purkis PE, Gardner ID, Van Muijen GNP, Lane EB. Site variation in keratin expression in human oral epithelia-an immunocytochemical study of individual keratins. Epithelial Cell Biol 1987;1:31-43.

11. Mackenzie IC, Rittman G, Gao Z, Leigh IM, Lane EB. Patterns of cytokeratin expression in human gingival epithelia. $J$ Periodont Res $1991 ; 26: 468-478$.

12. Morgan PR, Johnson NW, Leigh IM, Lane EB. Structure of gingival 
epithelium as revealed by monoclonal antibodies to keratins. In: Lehner T, Cimasoni I, eds. Borderland Between Caries and Periodontal Disease, vol. 3. London: Academic Press; 1986:47-59.

13. Steffensen B, Lopatin DE, Caffesse RG, Hanks CT. Blood group substances as differentiation markers in human dento-gingival epithelium. J Periodont Res 1987;22:451-455.

14. Juhl M, Reibel J, Stoltze K. Immunohistochemical distribution of keratin proteins in clinically healthy human gingival epithelia. Scand $J$ Dent Res 1989;97:159-170.

15. Mackenzie IC, Dabelsteen E, Mandel U. Expression of blood group antigen-related carbohydrates by human gingival epithelia. $J$ Periodont Res 1989;24:289-297.

16. Crawford JM, Hopp B. Junctional epithelium expresses the intercellular adhesion molecule ICAM-1. J Periodont Res 1990;25:254256.

17. Ten Cate AR. The gingival junction. In: Branemark PI, Zarb GA, Albrektsson T, eds. Tissue Integrated Prostheses. Chicago: Quintessence; 1985:145-153.

18. Meffert R. Implant therapy. In: Proceedings of the World Workshop in Clinical Periodontics. Chicago: American Academy of Periodontology; 1989:Section VIII.1-19.

19. Berglundh T, Lindhe J, Ericsson I, Marinello CP, Liljenberg B, Thomsen P. The soft tissue barrier at implants and teeth. Clin Oral Impl Res 1991;2:81-90.

20. Listgarten MA, Lang NP, Schroeder HE, Schroeder A. Periodontal tissues and their counterparts around endosseous implants. Clin Oral Impl Res 1991;2:1-19.

21. Buser D, Weber HP, Donath K, et al. Soft tissue reactions to nonsubmerged unloaded titanium implants in beagle dogs. J Periodontol 1992;63:226-236.

22. Marks SC, McKee MD, ZalZal S, Nanci A. The epithelial attachment and the dental junctional epithelium: Ultrastructural features in porcine molars. Anat Rec 1994;238:1-14.

23. Carmichael RP, McCulloch CAG, Zarb GA. Quantitative immunohistochemical analysis of keratins and desmoplakins in human gingiva and peri-implant mucosa. J Dent Res 1991;70:889-905.

24. James RA, Schultz RL. Hemidesmosomes and the adhesion of junctional epithelial cells to metal implants. J Oral Implantol 1973;4: 294-302.

25. Listgarten MA, Lai $\mathrm{CH}$. Ultrastructure of the intact interface between an endosseous epoxy resin dental implant and the host tissues. J Biol Buccale 1975;3:13-28.

26. McKinney RV, Steflik DE, Koth DL. Evidence for junctional epithelial attachment to ceramic dental implants, a transmission electron microscopic study. J Periodontol 1985;56:579-591.

27. Gould TR, Brunette DM, Westbury L. The attachment mechanism of epithelial cells to titanium in vitro. J Periodont Res 1981;16:611616.

28. Winter GD. Epidermal regeneration studied in the domestic pig. In: Maibach H, Rovee DE, eds. Epidermal Wound Healing. Chicago: Year Book; 1972

29. Mackenzie IC, Dabelsteen E, Zimmerman K. The relationship between expression of epithelial B-like blood group antigen, cell movement and cell proliferation. APMIS 1977;85:49-56.

30. Mackenzie IC. Formation and sequential changes of the periodontium. In: Genco RJ, Goldman HM, Cohen DW, eds. Contemporary Periodontics. St. Louis: The CV Mosby Company; 1989:55-62.

31. Mackenzie IC. Factors influencing the stability of the gingival sulcus. In: Guggenheim B, ed. Periodontology Today. Basel: Karger; 1988:41-49.

32. Tonetti MS, Gerber L, Lang NP. Vascular adhesion molecules and initial development of inflammation in clinically healthy human keratinized mucosa around teeth and osseointegrated implants. $J$ Periodont Res 1994;29:386-392.

33. Tonetti MS, Schmid J, Hammerle CH, Lang NP. Intraepithelial an- tigen-presenting cells in the keratinized mucosa around teeth and osseointegrated implants. Clin Oral Impl Res 1993;4:37-48.

34. Mackenzie IC, Gao Z. Patterns of cytokeratin expression in the epithelia of inflamed human gingiva and periodontal pockets. J Periodont Res 1993;28:49-59.

35. Listgarten MA. Ultrastructure of the dento-gingival junction after gingivectomy. J Periodont Res 1972;7:151-160.

36. Braga AM, Squier CA. Ultrastructure of regenerating junctional epithelium in the monkey. $J$ Periodontol 1980;51:386-392.

37. Schulz J, Ermich T, Kasper M, Raabe G, Schumann D. Cytokeratin pattern of clinically intact and pathologically changed oral mucosa. Int J Oral Maxillofac Surg 1992;1:35-39.

38. Bosch FX, Ouhayoun JP, Bader BL, et al. Extensive changes in cytokeratin expression patterns in pathologically affected human gingiva. Virchows Archiv B (Cell Pathol) 1989;58:59-77.

39. Mackenzie IC. Epithelial-mesenchymal interactions in the development and maintenance of epithelial tissues. In: Lane EB, Leigh IM, Watt FM, eds. The Keratinocyte Handbook. Cambridge: Cambridge University Press; 1994:243-258.

40. Perkins W, Campbell I, Leigh IM, Mackie RM. Keratin expression in normal skin and epidermal neoplasms. J Cutan Pathol 1992;49: 476-482.

41. Gao Z, Mackenzie IC. Patterns of phenotypic expression of human junctional, gingival and reduced enamel epithelia in vivo and in vitro. Epithelial Cell Biol 1992;1:156-167.

42. Hertle MD, Kubler MD, Leigh IM, Watt FM. Aberrant integrin expression during epidermal wound healing and in psoriatic epidermis. $J$ Clin Invest 1992;6:1892-1901.

43. Hormia M, Virtanen I, Quaranta V. Immunolocalization of integrin $\alpha_{6} \beta_{4}$ in mouse junctional epithelium suggests an anchoring function to both the internal and the external basal lamina. $J$ Dent Res 1992;71:1503-1508.

44. Gao Z, Mackenzie IC, Pan S, Shi J. Epithelial lining of sinus tracts associated with periapical disease: An immunocytochemical study using monoclonal antibodies to keratins. J Oral Pathol Med 1991;20:228-233.

45. Listgarten MA. Similarity of epithelial relationships in the gingiva of rat and man. $J$ Periodontol 1975;46:677 -680.

46. Yamasaki A, Nikai H, Niitani K, Ijuhin N. Ultrastructure of the junctional epithelium of germfree rat gingiva. $J$ Periodontol 1979;50:641-648.

47. Kasper M, Karsten U, Stosiek P, Moll R. Distribution of intermediate-filament proteins in the human enamel organ: unusually complex pattern of coexpression of cytokeratin polypeptides and vimentin. Differentiation 1989;40:207-217.

48. Gao Z, Mackenzie IC, Cruchley AT, Williams DM, Leigh IM, Lane EB. Cytokeratin expression of the odontogenic epithelia in dental follicles and developmental cysts. J Oral Pathol Med 1989;18:6367.

49. Pelissier A, Ouhayoun JP, Sawaf MH, Forest N. Changes in cytokeratin expression during the development of the human oral mucosa. J Periodont Res 1992;27:588-598.

50. Toh H, Rittman G, Mackenzie IC. Keratin expression in taste bud cells of the circumvallate and foliate papillae of adult mice. Epithelial Cell Biol 1993;2:126-133.

51. Salonen JI, Kantsky MB, Dale BA. Changes in cell phenotype during regeneration of junctional epithelium of human gingival in vitro. J Periodont Res 1989;24:370-377.

52. Verdickt GM, Savage NW, Dodd NM, Walsh LJ. Expression of the CD54 (ICAM-1) and CD11a (LFA-1) adhesion molecules in oral mucosal inflammation. J Oral Pathol Med 1992;21:65-69.

53. Gemmell E, Walsh LJ, Savage NW, Seymour GJ. Adhesion molecule expression in chronic inflammatory periodontal disease tissue. $J$ Periodont Res 1994;29:46-53.

54. Crawford JM. Distribution of ICAM-1, LFA-3 and HLA-Dr in 
healthy and diseased gingival tissues. J Periodont Res 1992;27:291298.

55. Mackenzie IC, Hill MW. Connective tissue influences on patterns of epithelial architecture and keratinization. Cell Tissue Res 1984;235: 551-559.

56. Doran TI, Vidrich A, Sun TT. Intrinsic and extrinsic regulation of the differentiation of skin, corneal and esophageal epithelial cells. Cell 1980;22:17-25.

57. Saxen L. Directive versus permissive induction: A working hypothesis. In: Lash JW, Burger MM, eds. Cell and Tissue Interactions. New York: Raven Press; 1977.

58. Sengel P. Morphogenesis of Skin. Cambridge: Cambridge University Press; 1976.

59. Kedinger M, Simon-Assmann P, Haffen K. Growth and differentiation of intestinal endodermal cells in a coculture system. Gut 1987;28:237-241.

60. Cunha GR, Young P, Hamamoto S, Guzman R, Nandi S. Developmental response of adult mammary epithelial cells to various fetal and neonatal mesenchyme. Epithelial Cell Biol 1992;1:105-118.

61. Mackenzie IC, Rittman G, Bohnert A, Breikreutz D, Fusenig NE. Influence of connective tissues on the in vitro growth and differentiation of murine epidermis. Epithelial Cell Biol 1993;2:107-119.

62. Boukamp P, Breitkreutz D, Stark HJ, Fusenig NE. Mesenchymemediated and endogenous regulation of growth and differentiation of human skin keratinocytes derived from different body sites. Differentiation 1990;44:150-161.
63. Fusenig NE, Breitkreutz D, Boukamp P, Bohnert A, Mackenzie IC. Epithelial-mesenchymal interactions in tissue homeostasis and malignant transformation. In: Johnson NW, ed. Risk Markers of Oral Disease, vol. 2. Cambridge: Cambridge University Press; 1991:218256.

64. Mackenzie IC, Hill MW. Maintenance of regionally specific patterns of cell proliferation and differentiation in transplanted skin and oral mucosa. Cell Tissue Res 1981;219:597-607.

65. Holmstrup P, Dabelsteen E, Stoltze K, Worsaae N. Epithelial outgrowths from human buccal mucosa transplants in nude mice. Scand $J$ Dent Res 1981;89:417-423.

66. Rubin JS, Osada H, Finch PW, Taylor WG, Rudikoff S, Aaronson SA. Purification and characterization of a newly identified growth factor specific for epithelial cells. Proc Natl Acad Sci (USA) 1989;86:802-806.

67. Werner S, Peters KG, Longaker MT, Fuller-Pace F, Banda MJ, Williams LT. Large induction of keratinocyte growth factor expression in the dermis during wound healing. Proc Natl Acad Sci (USA) - 1992;89:6896-6900.

68. Mackenzie IC. Nature and mechanisms of regeneration of the junctional epithelial phenotype. J Periodontol 1987;22:243-245.

Send reprint requests to: Dr. Ian Mackenzie, 2361 Dent 1078, School of Dentistry, University of Michigan, Ann Arbor, MI 48109-1078.

Accepted for publication April 20, 1995. 